\title{
Emprego do Método UTD/MoM para Análise de Antena Helicoidal Instalada em Aeronaves
}

\author{
R.R. de Assis, I. Bianchi
}

\begin{abstract}
Resumo - Neste trabalho é analisado o efeito causado pela estrutura de uma aeronave de médio porte nas características de irradiação de uma antena helicoidal. A análise é feita utilizando o método híbrido UTD/MoM do SuperNEC na faixa de freqüência de comunicação via satélite. Os resultados são apresentados em gráficos e consolidados em tabela.
\end{abstract}

Palavras-Chave - Antena Helicoidal, Teoria Uniforme da Difração (UTD), Método dos Momentos (MoM), SuperNEC, aeronave.

Abstract - In this work it is analyzed the effect caused by the structure of a medium sized aircraft on the radiation features of a helical antenna. The analysis is performed using the SuperNEC UTD/MoM hybrid method at the satellite communication frequency band. The results are presented in graphs and consolidated in table.

Index Terms- Helix Antenna, Uniform Theory of Diffraction (UTD), Method of Moments (MoM), SuperNEC, aircraft.

\section{INTRODUÇÃO}

A comunicação de uma aeronave com satélite é atualmente um serviço indispensável na aviação. Para atender os requisitos dos diversos sistemas existentes, há uma variedade de antenas, com diferentes tecnologias de construção. Para projeto e análise destas antenas, dispõem-se atualmente de simuladores eletromagnéticos que empregam técnicas numéricas variadas, sendo estas aproximadas ou rigorosas [1, 2].

Quando antenas são instaladas sobre estruturas complexas, tais como aeronaves, é imprescindível o emprego destes simuladores, pois estes possibilitam o cálculo dos efeitos da estrutura da aeronave sobre as características de irradiação da antena.

Com objetivo de analisar estes efeitos, existe uma variedade de abordagens na literatura [3]-[9]. Sabe-se também que há uma limitação computacional no emprego de determinadas técnicas numéricas para analisar estruturas eletricamente grandes. Desta forma, é proposta a utilização da técnica híbrida que engloba a teoria de difração uniforme (UTD) e o método dos momentos (MoM) [10, 11].

Utilizando o simulador SuperNec [12], são analisados neste artigo os efeitos das asas, fuselagem, motores e empenagem sobre as figuras de mérito de uma antena helicoidal, projetada para operar na faixa de freqüência de comunicação via satélite e instalada sobre uma aeronave de médio porte. O conhecimento destes efeitos é importante, pois possibilita escolher o melhor posicionamento da antena sobre a aeronave.

R.R. de Assis e I. Bianchi, Laboratório de Antenas e Propagação - LAP, Instituto Tecnológico de Aeronáutica, São José dos Campos, SP, E-mails: r2.assis@gmail.com, ibianchi@ita.br.

\section{TEORIA}

Os efeitos produzidos nas características de irradiação de uma antena instalada sobre estruturas complexas, são atribuídos às múltiplas reflexões e difrações sofridas pela onda eletromagnética em seu percurso. Para computar os efeitos devido às próprias estruturas, o método híbrido UTD/MoM do SuperNEC modela a antena através do MoM e insere as possíveis alterações no cálculo do campo total, devidos às reflexões e difrações de outras estruturas próximas, utilizando o método da teoria da difração uniforme.

A idéia fundamental da técnica híbrida usando MoM/UTD é modificar a matriz impedância obtida do MoM de forma a incluir os efeitos calculados pelo método UTD [11]. Sendo assim os elementos da nova matriz $\left[Z^{\prime}\right]$ serão,

$$
Z_{m n}^{\prime}=Z_{m n}+Z_{m n}^{U T D}
$$

onde, os elementos representados por $Z_{\mathrm{mn}}$ são obtidos das soluções das integrais de Pocklington [13] pelo MoM e os elementos representados por $Z_{m n}^{U T D}$ são as contribuições devido às difrações e reflexões obtidas da utilização do método UTD.

Assim, os coeficientes para determinar a densidade de corrente sobre o elemento irradiador devido às contribuições de campo calculadas pelos métodos MoM e UTD são obtidos da solução da equação matricial

$$
\left[I^{\prime}\right]=\left[Z^{\prime}\right]^{-1}[V]
$$

sendo [ $V]$ o vetor excitação.

A técnica híbrida apresenta-se vantajosa do ponto de vista computacional, pois possibilita ter uma boa precisão na distribuição de corrente em regiões próximas à fonte de energia através do MoM. Por outro lado, apesar da precisão ser menor nas regiões distantes da fonte quando se emprega o método UTD, os resultados não são comprometidos. Essa vantagem é ressaltada quando se deseja analisar estruturas eletricamente grandes, onde as técnicas rigorosas tornam-se inviáveis computacionalmente.

Componentes relevantes de campo calculadas pelo método UTD são ilustradas na Fig. 1, sendo estas devido às difrações ocorridas nas bordas, nos cantos e as reflexões, conforme mostrado em [14]. No SuperNEC tem-se o recurso da escolha dos efeitos a serem computados. Sendo assim, é possível isolar os efeitos devido aos campos refletidos, aos campos difratados e aos campos diretos, podendo com isso, identificar e avaliar o efeito causado pelas estruturas UTD existentes no modelo. 


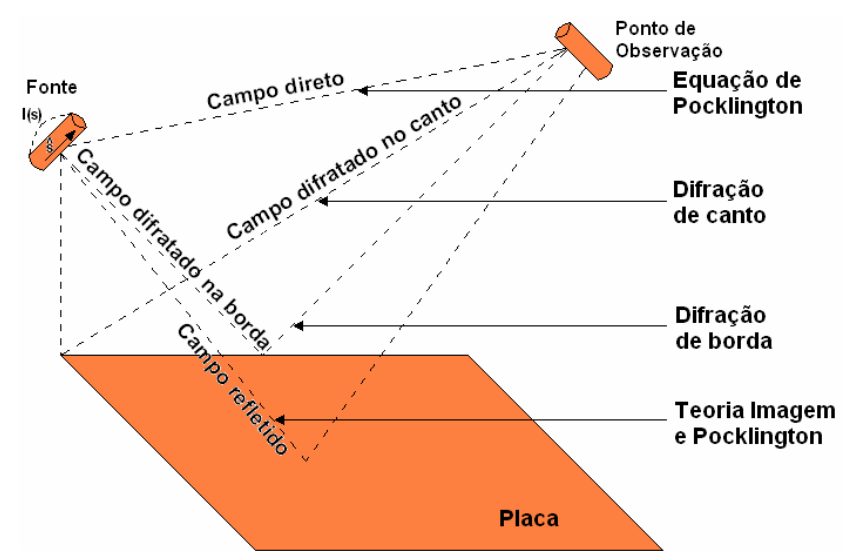

Fig. 1. Componentes de campo consideradas no método UTD .

\section{ANTENA HELICOIDAL COM PLACA CONDUTORA}

Com objetivo de visualizar os efeitos causados por estruturas metálicas nas características de irradiação de uma antena helicoidal, posicionou-se a mesma em $x=-1,414 \mathrm{~m}$, $y=0 \mathrm{~m}$ e $z=1,414 \mathrm{~m}$, na presença de uma placa condutora perfeita de dimensões $1 \mathrm{mx} 1 \mathrm{~m}$, conforme ilustra a Fig. 2 . Considerando a teoria de antena helicoidal [13] e com auxílio do SuperNEC para a análise, a antena é projetada para operar na freqüência de $1600 \mathrm{MHz}$, com polarização circular para a direita no modo axial e ângulo de apontamento $\phi=0^{\circ}$ e $\theta=90^{\circ}$. Nestas condições obteve-se uma impedância de entrada $Z_{I N} \approx 178,1+\mathrm{i} 44,4[\Omega]$, largura de feixe $H P B W \approx 44^{\circ}$ no plano $x z$, diretividade $\mathrm{D}_{0} \approx 11,4 \mathrm{~dB}$ e a razão axial $\mathrm{RA} \approx 0,57 \mathrm{~dB}$ em $\phi=0^{\circ}$ e $\theta=90^{\circ}$. Utilizou-se 7 espiras, com diâmetro de 5,968 cm, espaçamento entre espiras de 4,33 cm, raio do elemento irradiador de $1 \mathrm{~mm}$ sendo o comprimento total de $30,3 \mathrm{~cm}$. A antena foi considerada sem perdas com um plano de terra com dimensões de $25 \mathrm{~cm}$ x $25 \mathrm{~cm}$.



Fig. 2. Estrutura proposta para a análise (polarização circular).

Para computar os efeitos da placa, foi utilizado o método UTD do SuperNEC, onde foram considerados somente os efeitos relacionados ao campo direto e ao campo refletido. São simuladas 3 condições distintas, (1) considerando somente o campo direto, (2) considerando somente o campo refletido e (3) considerando campo direto e campo refletido.

Nas Figs. 3 (a), (b) e (c) são mostrados os resultados de campo elétrico no plano $z=2 \mathrm{~m}$ considerando as condições de simulação (1), (2) e (3) respectivamente. A Fig. 3 (c) mostra o efeito produzido pelo campo refletido na placa nas linhas de campo em uma região do plano $z=2 \mathrm{~m}$. A Fig. 3 (d) ilustra o diagrama de irradiação tridimensional da antena, onde são observados os efeitos atribuídos à presença da placa condutora.



(a)

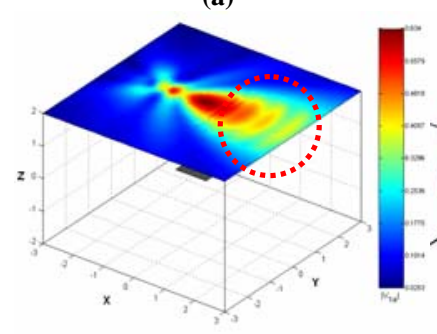

(c)



(b)
Fig. 3. Resultados da antena helicoidal com placa condutora, (a) Campo elétrico em uma região no plano $z=2 \mathrm{~m}$ na condição 1 (campo direto); (b) Campo elétrico em uma região no plano $z=2 \mathrm{~m}$ na condição 2 (campo refletido); (c) Campo elétrico em uma região no plano $z=2 \mathrm{~m}$ na condição3 (campos direto e refletido); (d) Diagrama de irradiação 3D.

Para efeito de clareza, a Fig. 4 apresenta o diagrama no plano $x z$, onde é possível notar entre os ângulos de $30^{\circ}$ e $60^{\circ} \mathrm{o}$ efeito produzido pela reflexão e entre os ângulos de $120^{\circ}$ e $150^{\circ}$, os efeitos que a placa causa ao obstruir o campo irradiado pela antena.

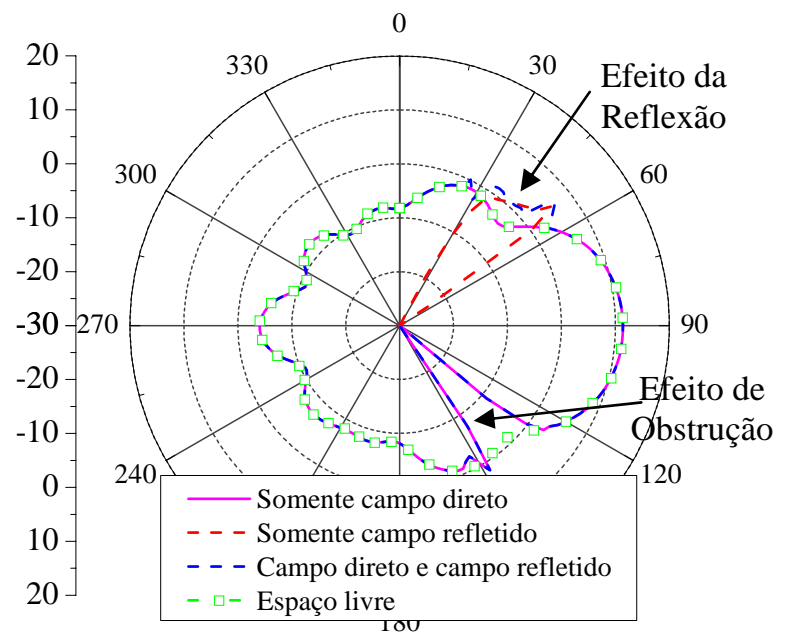

Fig. 4. Diagrama de irradiação no plano $x z$.

A Fig. 5 ilustra razão axial versus ângulo de elevação para o plano $x z$. São mostradas as curvas obtidas com a antena na ausência da placa condutora (curva azul) e a antena na presença da placa (curva vermelha). Observa-se claramente que entre os ângulos de $20^{\circ}$ e $60^{\circ}$ a razão axial sofre uma alteração devido ao campo refletido na placa. Observa-se também o efeito relacionado com a obstrução do campo pela placa condutora. A 
razão axial em $\phi=0^{\circ}$ e $\theta=90^{\circ}$ permanece sendo $0,57 \mathrm{~dB}$.

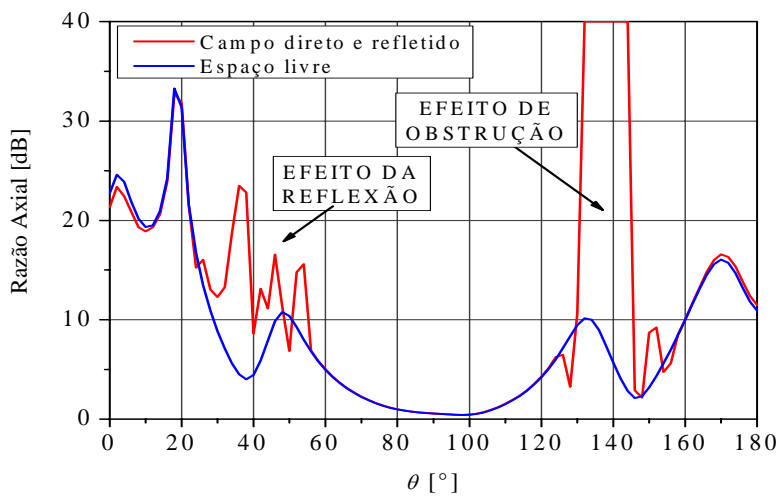

Fig. 5. Razão axial da antena helicoidal com e sem placa condutora.

\section{ANTENA HELICOIDAL COM AERONAVE}

Neste item são analisadas as características de irradiação da antena helicoidal descrita na seção III, na presença de uma aeronave de médio porte.

A aeronave possui um comprimento de aproximadamente $30 \mathrm{~m}$, uma envergadura de $20 \mathrm{~m}$ e diâmetro da fuselagem de 2,28 m, cujo modelo eletromagnético foi construído no SuperNEC, conforme ilustra a Fig. 6.

A abordagem consiste em analisar os efeitos resultantes devido à reflexão e à difração, produzidas por partes da estrutura da aeronave considerando três possíveis posições de instalação da antena, conforme mostra a Fig. 6, ou seja, a posição 1 sendo próxima à cabine dos pilotos, a posição 2 próxima às asas e a posição 3 próxima aos motores. Ressalta-se que são desconsiderados os demais efeitos, como por exemplo, interferências entre sistemas.

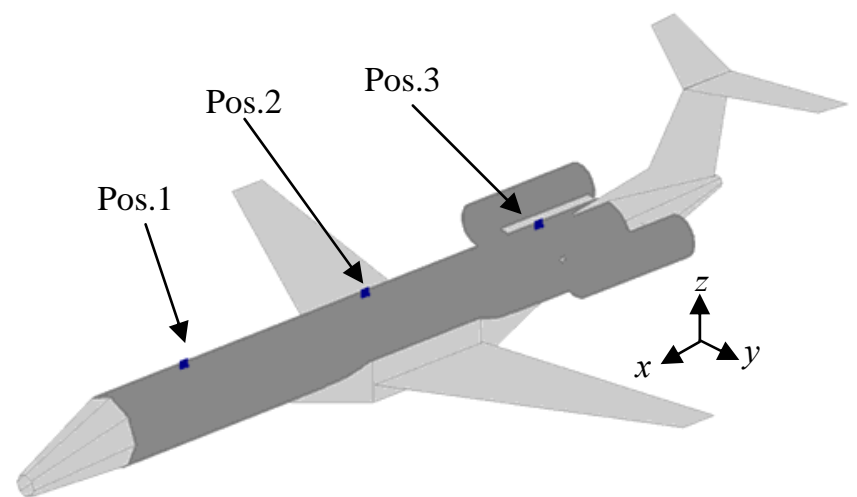

Fig. 6. Modelo eletromagnético UTD, SuperNEC.

Para analisar os efeitos produzidos pelas partes da aeronave os seguintes modelos foram considerados:

- Modelo completo da aeronave (Fig. 6).

- Modelo [F], contendo apenas a fuselagem (Fig. 7 (a)).

- Modelo [F+A], contendo a fuselagem e as asas (Fig. 7 (b)).

- Modelo [F+M], contendo a fuselagem e os motores (Fig. 7 (c)).

- Modelo [F+E], contendo a fuselagem e a empenagem (Fig. 7 (d)).

- Modelo [F+A+M+E], contendo a fuselagem, asas, motores e empenagem (Fig. 7 (e)).



(a)

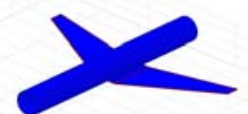

(b)



(c)

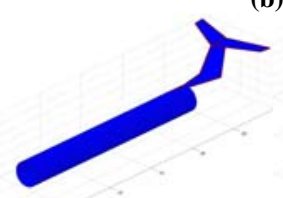

(d)

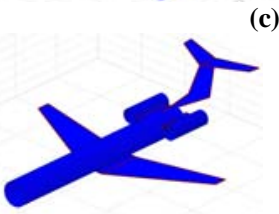

(e)
Fig. 7. Partes da estrutura da aeronave simuladas: (a) fuselagem [F]; (b) fuselagem e asas [F+A]; (c) fuselagem e motores [F+M]; (d) fuselagem e empenagem [F+E] (e) fuselagem, asas, motores e empenagem $[\mathrm{F}+\mathrm{A}+\mathrm{M}+\mathrm{E}]$.

Considerando que esta antena pode assumir diferentes direções de apontamento, escolheu-se aqui a condição que o eixo da hélice é direcionado para $\phi=90^{\circ}$ e $\theta=85^{\circ}$, conforme ilustra a Fig. 8. Esta condição pode ser considerada crítica para antenas instaladas em aeronaves $[15,16]$.

A Fig. 8 mostra também o detalhe do posicionamento da antena sobre a aeronave, onde não há contato entre o plano de terra e a fuselagem, sendo esta distância aproximadamente $0,1 \lambda$.

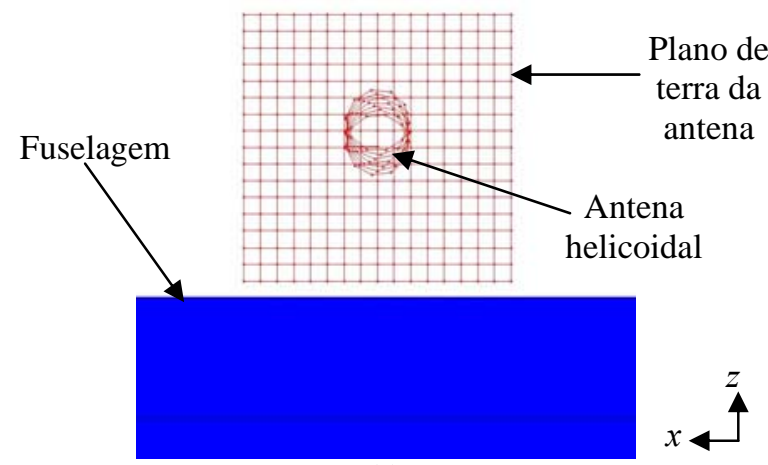

(a)

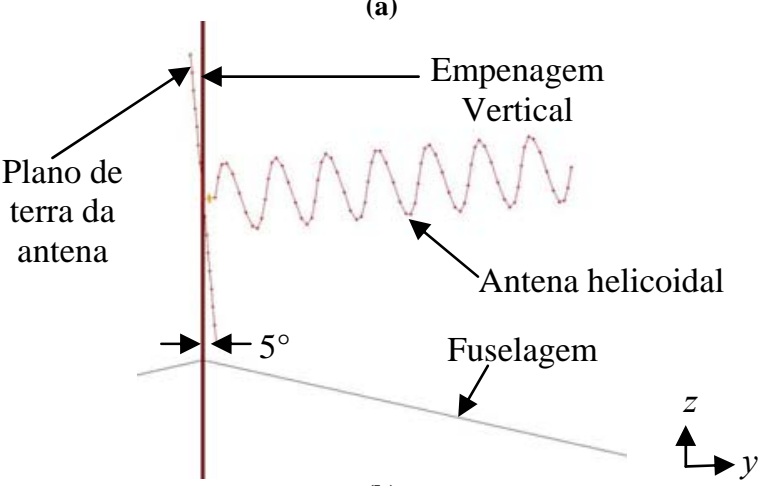

(b)

Fig. 8. Detalhe do posicionamento da antena na aeronave, (a) vista lateral, (b) vista frontal.

Para as diferentes localizações da antena conforme ilustra a Fig. 6 são consideradas as seguintes condições de análise: (1) "somente campo direto", onde os efeitos de campo refletido e difratado são suprimidos, (2) "sem campo direto", condição em que o efeito do campo direto é suprimido e (3) "com efeitos completos”, ou seja, estão presentes os efeitos dos campos refletidos, difratados e do campo direto. As Figs. 9 a 12 apresentam os diagramas de irradiação resultantes de algumas simulações na condição de análise (3). 
A Fig. 9 mostra o diagrama de irradiação da antena na posição 1, sendo que a Fig. 9 (a) é o resultado considerando o Modelo [F] e a Fig. 9 (b) o resultado considerando o Modelo [F+A+M+E]. Neste caso houve pouca influência da estrutura da aeronave sobre o feixe principal da antena. A Fig. 10 representa o diagrama de irradiação da antena na posição 2, sendo a Fig. 10 (a) o resultado utilizando o Modelo [F] e a Fig. 10 (b) o resultado utilizando o Modelo [F+A]. A influência da asa é evidenciada pelas ondulações apresentadas no feixe principal do diagrama de irradiação (Fig. 10 (b)). A Fig. 11 apresenta o diagrama de irradiação da antena na posição 3, sendo a Fig. 11 (a) o resultado que considera o Modelo [F] e a Fig. 11 (b) o resultado que considera o Modelo $[\mathrm{F}+\mathrm{M}]$. Neste caso a influência dos motores sobre o diagrama de irradiação é bem maior devido à proximidade dos motores com antena.

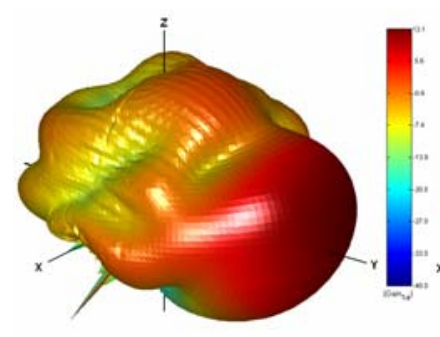

(a)

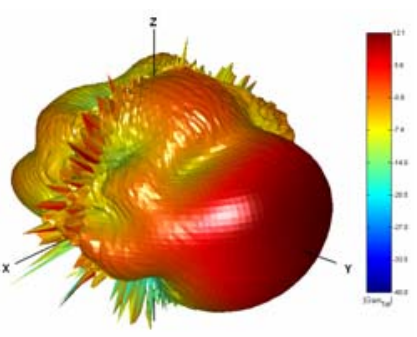

(b)
Fig. 9. Diagrama de irradiação 3D para a antena localizada na posição 1: (a) modelo [F]; (b) modelo [F+A+M+E].

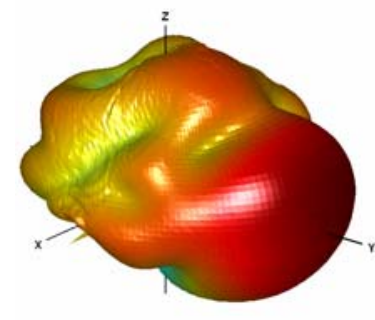

(a)

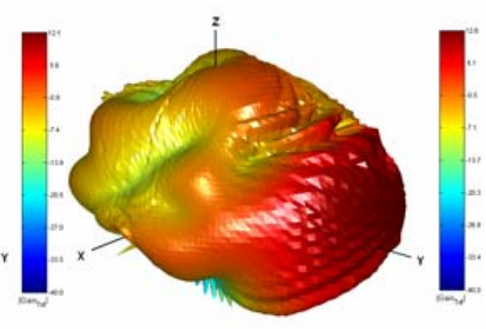

(b)
Fig. 10. Diagrama de irradiação 3D para a antena localizada na posição 2: (a) modelo [F]; (b) modelo [F+A].

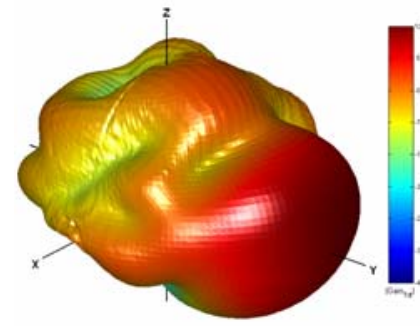

(a)



(b)
Fig. 11. Diagrama de irradiação 3D para a antena localizada na posição 3: (a) modelo [F]; (b) modelo [F+M].

A Fig. 12 mostra cortes de diagramas de irradiação no plano $y z$ considerando o Modelo [F+A+M+E], para as condições de análise (1), (2) e (3), considerando as antenas localizadas nas três posições propostas. Nesta figura pode ser observado o efeito dos campos difratados e refletidos na composição do diagrama completo. Estes efeitos se tornam mais pronunciados à medida que a antena é aproximada das asas e dos motores, como pode ser observado nas Figs. 12 (b) e (c). Observa-se também que ocorre um desvio no lóbulo principal da antena, da ordem de $10^{\circ}$, onde este desvio é atribuído ao fato da antena ser projetada na ausência da estrutura da aeronave. Observa-se nas Figs 12 (b) e 12 (c) uma queda acentuada nas curvas que consideram somente o campo direto (entre $90^{\circ}$ e $120^{\circ}$ ). Este fenômeno é atribuído à obstrução do campo devido às asas e aos motores.

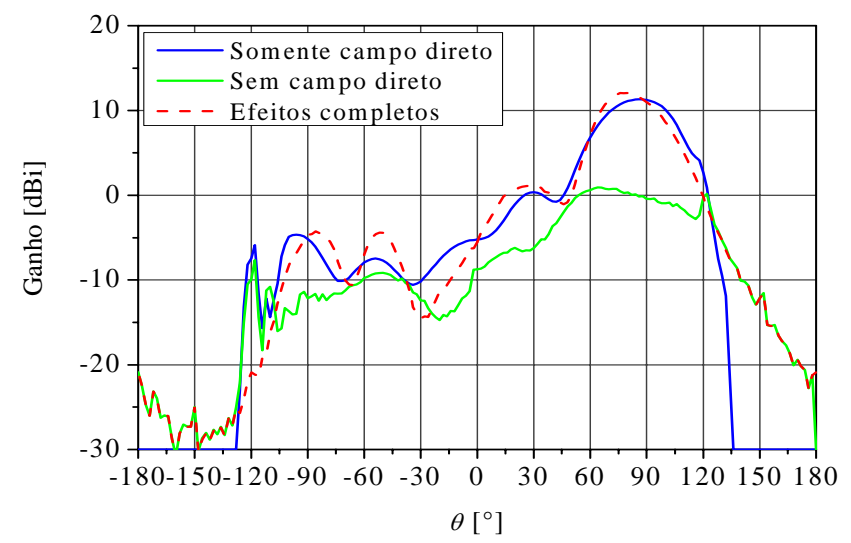

(a)

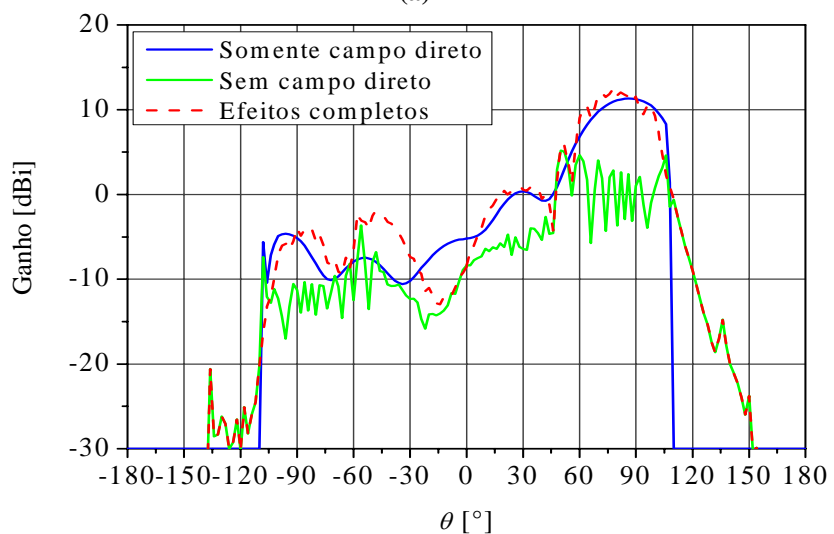

(b)



(c)

Fig. 12. Diagrama de irradiação no plano $y z$, Modelo [F+A+M+E], (a) antena na posição 1, (b) antena na posição 2, (c) antena na posição 3.

Na Tabela I são agrupados os resultados das simulações em $1600 \mathrm{MHz}$ na condição de análise (3). Observa-se que a impedância de entrada da antena sofreu uma pequena variação ao ser posicionada sobre a fuselagem, em torno de $1,2 \%$ na parte real. A inclusão das demais partes da aeronave não contribuiu significativamente para a mudança no valor da impedância. 
A diretividade teve uma variação em torno de $0.9 \%$ a $10 \%$ com relação à antena no espaço livre. A asa é a parte da estrutura que mais influencia quando a antena é localizada na posição 2. Isto pode ser atribuído ao fato de que, na direção do lóbulo principal da antena e nesta condição de apontamento, a asa possui uma área bem maior em relação às outras partes da estrutura e assim os efeitos de reflexão são mais significativos.

A largura de feixe no plano $y z$ também sofreu variação com relação ao espaço livre. A variação máxima ficou em torno de $6^{\circ}$ (13,6 \%) provocando a diminuição da largura de feixe, sendo que novamente a fuselagem teve a maior influência. $\mathrm{O}$ valor de $22^{\circ}$ contido na Tabela I em alguns casos está relacionado ao efeito de obstrução dos campos devido aos motores.

A razão axial em $\phi=90^{\circ}$ e $\theta=85^{\circ}$ também variou em função da inclusão das partes que compõem a estrutura. Os resultados mostrados na tabela indicam que a degradação observada da razão axial para a antena nas posições 1,2 e 3 é devida à fuselagem, asas e motores, respectivamente.

TABELA I

RESUlTADOS DAS SiMULAÇÕES EM 1600 MHz

\begin{tabular}{|c|c|c|c|c|c|}
\hline Modelo $^{1}$ & Pos. & $\begin{array}{c}\operatorname{Re}\left\{\mathrm{Z}_{\mathrm{IN}}\right\} \\
{[\Omega]} \\
\end{array}$ & $\begin{array}{c}\mathrm{D}_{0}{ }^{2} \\
{[\mathrm{dBi}]} \\
\end{array}$ & $\begin{array}{c}\mathrm{HPBW}^{3} \\
\left.{ }^{\circ}\right] \\
\end{array}$ & $\begin{array}{l}\mathrm{RA}^{2} \\
{[\mathrm{~dB}]} \\
\end{array}$ \\
\hline E.Livre & - & 178,1 & 11,4 & 44 & 0,57 \\
\hline $\mathrm{F}$ & 1 & 180,4 & 11,7 & 38 & 4,08 \\
\hline $\mathrm{F}+\mathrm{A}$ & 1 & 180,4 & 11,7 & 38 & 4,06 \\
\hline $\mathrm{F}+\mathrm{M}$ & 1 & 180,4 & $\begin{array}{l}11,7 \\
\end{array}$ & 38 & 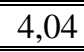 \\
\hline $\mathrm{F}+\mathrm{E}$ & 1 & 180,4 & 11,7 & 38 & 4,08 \\
\hline $\mathrm{F}+\mathrm{A}+\mathrm{M}+\mathrm{E}$ & 1 & 180,3 & 11,7 & 38 & 4,04 \\
\hline $\mathrm{F}$ & 2 & 180,1 & 11,7 & 38 & 4,09 \\
\hline $\mathrm{F}+\mathrm{A}$ & 2 & 180,1 & 12,2 & 40 & 4,43 \\
\hline $\mathrm{F}+\mathrm{M}$ & 2 & 180,1 & 11,7 & 38 & 4,02 \\
\hline $\mathrm{F}+\mathrm{E}$ & 2 & 180,1 & 11,7 & 38 & 4,09 \\
\hline $\mathrm{F}+\mathrm{A}+\mathrm{M}+\mathrm{E}$ & 2 & 180,1 & 12,2 & 40 & 4,38 \\
\hline $\mathrm{F}$ & 3 & 180,1 & 11,7 & 38 & 4,08 \\
\hline $\mathrm{F}+\mathrm{A}$ & 3 & 180,1 & 11,7 & 38 & 4,08 \\
\hline$\overline{F+M}$ & 3 & 180,3 & $\begin{array}{l}11,5 \\
\end{array}$ & 22 & 5,83 \\
\hline$\overline{\bar{F}+\mathrm{E}}$ & 3 & 180,1 & 111,7 & 38 & 4,08 \\
\hline $\mathrm{F}+\mathrm{A}+\mathrm{M}+\mathrm{E}$ & 3 & 180,3 & 11,5 & 22 & 5,83 \\
\hline
\end{tabular}

A Fig. 13 mostra os resultados da razão axial em $\phi=90^{\circ} \mathrm{e}$ $\theta=85^{\circ}$ e do coeficiente de onda estacionária de tensão $(V S W R)$ para o Modelo $[\mathrm{F}+\mathrm{A}+\mathrm{M}+\mathrm{E}]$ em função da freqüência. Observa-se que houve pouca variação do $V S W R$ na freqüência de operação em relação à antena no espaço livre. Entretanto, a razão axial varia consideravelmente em função da localização da antena. A norma RTCA DO-210D do Comitê Técnico de Rádio para Aeronáutica (RTCA) [17] estabelece padrões mínimos de desempenho operacional para serviços de satélite móvel aeronáutico. A análise mostra que a razão axial obtida para a antena localizada na posição 3 está no limiar do valor mínimo recomendado.

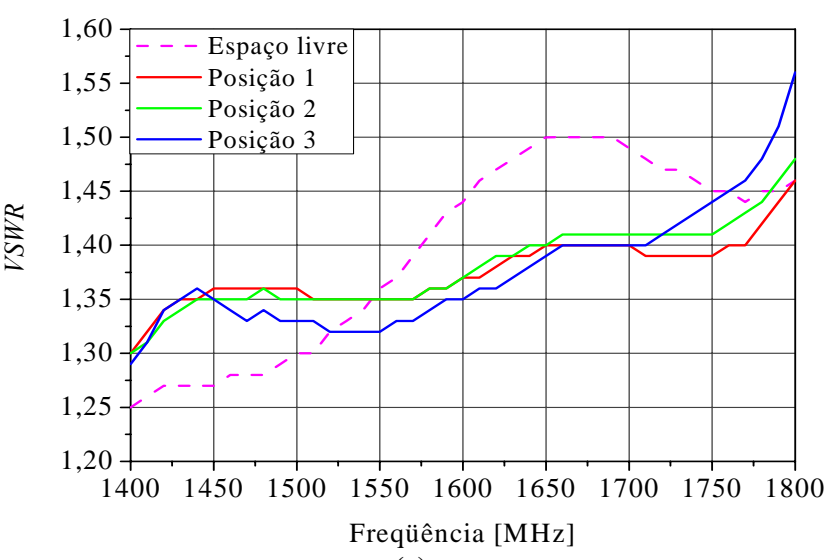

(a)

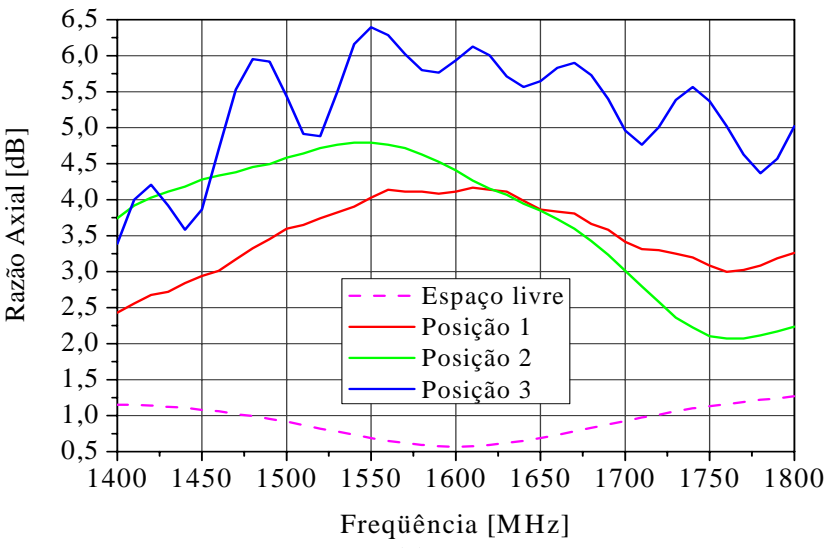

(b)

Fig. 13. Parâmetros da antena no modelo da aeronave $[\mathrm{F}+\mathrm{A}+\mathrm{M}+\mathrm{E}]$ : (a) $V S W R$, (b) Razão axial em $\phi=90^{\circ}$ e $\theta=85^{\circ}$.

A Fig. 14 ilustra o diagrama de irradiação no plano $y z$ da antena localizada na posição 2 , onde é possível relacionar o efeito de cada parte da aeronave com as alterações sofridas pelo diagrama de irradiação da antena. Observa-se que há sobreposição dos diagramas no intervalo de $0^{\circ}$ a $60^{\circ}$, para o Modelo [F+A+M+E] com o Modelo [F+M], concluindo-se que a alteração no diagrama é predominantemente devido aos motores. Conclusão análoga pode ser obtida comparando os diagramas do Modelo [F+A+M+E] com o Modelo [F+A], onde tem-se a contribuição das asas como predominante no intervalo entre $110^{\circ}$ e $150^{\circ}$.

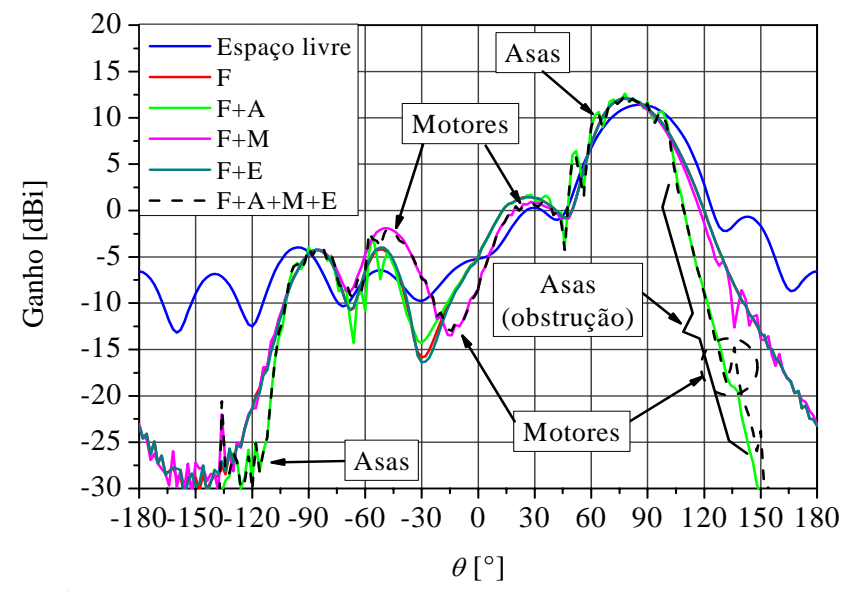

Fig. 14. Diagrama de irradiação no plano yz, modelo $[\mathrm{F}+\mathrm{A}+\mathrm{M}+\mathrm{E}]$, antena na posição 2 . 
A Fig. 15 compara os diagramas de irradiação obtidos no plano $y z$ considerando o Modelo completo da aeronave, e a antena localizada na três posições propostas. A antena localizada na posição 3 é a mais afetada pelos efeitos de reflexão e difração produzidos pela estrutura da aeronave, conforme observado na curva azul onde as oscilações são mais pronunciadas e o efeito de obstrução do diagrama é maior em torno de $90^{\circ}$ a $120^{\circ}$.

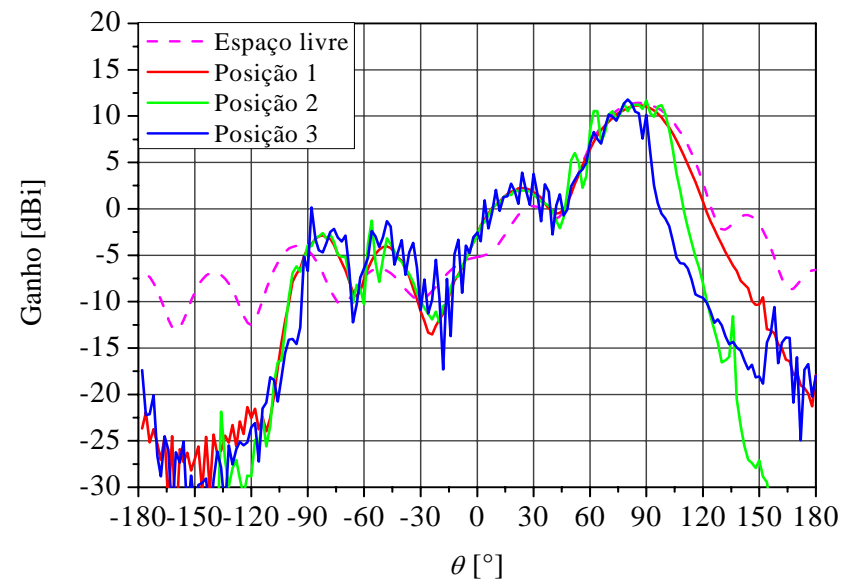

Fig. 15. Diagrama de irradiação no plano yz, modelo completo da aeronave.

Foram realizadas em torno de 50 simulações utilizando vários computadores, entre eles uma plataforma com 1 processador, 512 Mbytes de memória RAM operando com Windows XP de 32 bits e uma plataforma com 4 processadores, 8 Gbytes de memória RAM operando com Windows XP de 64 bits. O tempo computacional máximo estimado ficou em torno de 20 horas, usando a plataforma com 1 processador, e 10 horas, usando a plataforma com 4 processadores. Em ambos os casos a memória RAM requerida foi menor que aproximadamente 300 MBytes.

\section{CONCLUSÕES}

Neste artigo foi apresentado um estudo para analisar o efeito causado pela estrutura de uma aeronave de médio porte nas características de irradiação de uma antena helicoidal operando em $1600 \mathrm{MHz}$. Utilizou-se para análise o método híbrido UTD/MoM do SuperNEC.

Os resultados apresentados mostram que a razão axial e o diagrama de irradiação são as características da antena mais afetadas pela estrutura. Além disso, na condição proposta de direcionamento da antena em baixo ângulo de elevação, houve degradação no desempenho desejado devido às asas e aos motores, onde se conclui que a posição próxima à cabine dos pilotos da aeronave é a mais indicada para instalação de antenas de comunicação via satélite. Através do recurso de separação de efeitos de reflexões e difrações, disponível no método híbrido do SuperNEC, e com as simulações considerando apenas partes da estrutura, é possível identificar a parte da aeronave, responsável pela mudança na característica de irradiação da antena.

A memória RAM requerida pelo método híbrido UTD/MoM é pequena em comparação ao método MoM, pois esta, é diretamente relacionada com quantidade de segmentos utilizados para modelar a antena e a estrutura da aeronave, destacando-se assim a principal vantagem da utilização do método hibrido.

A metodologia apresentada foi satisfatória do ponto de vista de análise de posicionamento de antenas, onde foi possível identificar o efeito da estrutura sobre as características de irradiação da antena. Todavia o esforço de se utilizar técnicas de onda completa em toda a estrutura da aeronave ainda deve ser perseguido, visando aumentar a precisão dos resultados.

\section{REFERÊNCIAS}

[1] A. Hoorfar e V. Jamnejad, Electromagnetic Modeling and Analysis of Wireless Communication Antennas, IEEE Microwave Magazine, Vol. 4, p. 51-67, Março 2003.

[2] T,H, Hubing, Survey of Numerical Electromagnetic Modeling Techniques, University of Missouri-Rolla, Department of Electrical Engineering, Julho 1991.

[3] R.J. Marhefka e W.D. Burnside, Antennas on Complex Platforms, Proceedings of the IEEE, Vol. 80, No 1, p. 204-208, Janeiro 1992.

[4] A. Rubinstein, F. Rachidi, M. Rubinstein e B. Reusser, A Parallel Implementation of NEC for the Analysis of Large Structures, IEEE Transactions on Electromagnetic Compatibility, Vol. 45, No 2, p. 177188, 2003.

[5] G.F. Paynter, W.D. Burnside e T.H. Lee, A Systematic Approach to Design and Analysis of Antennas on Complex Platforms, IEEE Antennas and Propagation Magazine, Vol. 43, N 6, p. 38-44, Dezembro 2001.

[6] S.A. Davidson e G.A. Thiele, A Hybrid Method of Moments - GTD Technique for Computing Electromagnetic Coupling Between Two Monopole Antennas on a Large Cylindrical Surface, IEEE Transactions on Electromagnetic Compatibility, Vol. EMC-26, N² 2, p. 90-97, Maio 1984.

[7] R. Luebbers, R. Ohs e D. Carpenter, Positioning Antennas On Military Aircraft Using FDTD 3-D EM Analysis, RF Globalnet application note, Outubro 2006.

[8] S. Hausman, L. Januszkiewicz, Application of UTD, MoM, and FDTD to the Scattering Problem of a Finite PEC Cylinder, Radar Symposium 2006, IRS 2006, Krakow, Poland, p. 1-4, Maio 2006.

[9] M.V.T. Heckler, L.A. Greda e A. Dreher, Analysis of a Navigation Antenna Installed on a Civil Airplane, IEEE Antennas and Propagation Society International Symposium 2008, p. 1-4, Julho 2008.

[10]U. Jakobus e F.M. Landstorfer, A combination of Current- and Raybased Techniques for the Efficient Analysis of Electrically Large Scattering Problems, Proceedings of the 13th Annual Review of Progress in Applied Computational Electromagnetics, Vol. № 1, p. 746755, 1997.

[11]G.A. Thiele e T.H. Newhouse, A Hybrid Technique for Combining Moment Methods with the Geometrical Theory of Diffraction, IEEE Transactions on Antennas and Propagation, Vol. AP-23, $\mathrm{N}^{\circ}$ 1, Janeiro 1975.

[12]Poynting Software (Pty) Ltd., SuperNEC Versão 2.9, Disponível em www.supernec.com.

[13]C.A. Balanis, Antenna Theory Analysis and Design, Second Edition, John Wiley \& Sons Inc, 1997.

[14]A. Fourie e D. Nitch, SuperNEC: Antenna and Indoor-Propagation Simulation Program, IEEE Antennas and Propagation Magazine, Vol. 42, No 3, p. 31-48, Junho 2000.

[15]S.D. Ilcev, Airborne Satellite Antenna Mount and Tracking Systems, IEEE Africon 2004, Vol. 1, p. 289-295, Setembro 2004.

[16]J.W. Dahlgren e L.G. Darian, Narrowband Antenna Challenges for Airborne Platforms, Military Communications Conference 2001, MILCOM 2001, Vol. № 2, p. 1275-1277, IEEE 2001.

[17] Radio Technical Commission for Aeronautics (RTCA, Inc.), RTCA DO210D, http://www.rtca.org/. 